\title{
5 Twitter als Quelle wissenschaftlicher Analysen
}

Der Mikroblogging-Dienst Twitter ist seit einiger Zeit eine viel genutzte Quelle für sozial- und wirtschaftswissenschaftliche, aber auch technische Studien. „In addition to being a versatile communications platform to users around the globe, Twitter is also an excellent source of current information" (Gaffney \& Puschmann, 2014, S. 55). Der Dienst vereint also vielseitige Kommunikationsmöglichkeiten mit einer guten Verfügbarkeit von Informationen, wobei letzteres nicht nur für die eigentlichen Nutzer gilt, sondern auch für die Forschung. Dennoch hat Twitter auch zahlreiche Einschränkungen, zum Beispiel hinsichtlich der Qualität und Verlässlichkeit der Daten. Die folgenden Kapitel skizzieren die Vor- und Nachteile von Twitter-Daten für die Wissenschaft und bewerten die Eignung von Twitter als Quelle für wissenschaftliche Analysen anhand mehrerer Aspekte.

\subsection{Informationsgehalt}

Ein großer Vorteil von Twitter im Vergleich zu anderen sozialen Netzwerken wie Facebook oder MySpace ist die offene Kommunikation. Einzelne Meldungen/Tweets oder ganze Konversationen können durch jeden gesucht und gelesen werden - unabhängig, ob man als Nutzer registriert und mit einer Person befreundet ist. Diese offene Gestaltung schafft einen hohen Grad an Publizität (Jürgens \& Jungherr, 2011, S. 205). Dies kann hinsichtlich der häufig geführten DatenschutzDebatte ein Problem sein, ist jedoch für Forschende von großem Nutzen: Zum einen erhält man Zugriff auf umfassende und ungefilterte Daten, zum anderen müssen (zunächst) keine strengen Datenschutz- und Anonymisierungsauflagen eingehalten werden. Zudem können die Daten detailliert sein: Neben personenbezogenen Informationen, wie Name, Wohnort und Sprache, Followers und Followings, kann über die einzelnen Tweets, Retweets, Mentions und Favorites die Einstellung zu Themen und Nutzern oder die Stimmung abgefragt werden. Ferner stehen diese Informationen - bei Verwendung der frei zugänglichen Twitter APIs kostenlos zur Verfügung. Beim Sammeln und Verknüpfen dieser Daten über einen

(C) Der/die Autor(en) 2016

F. Pfaffenberger, Twitter als Basis wissenschaftlicher Studien,

DOI 10.1007/978-3-658-14414-2_5 
längeren Zeitraum erhält man eventuell umfassende Informationen über das Verhalten, die Stimmung oder Einstellung zu einem Thema.

Aufgrund der Begrenzung der Tweets auf 140 Zeichen und einer einfachen Gestaltung des Dienstes können Informationen schnell an einzelne Personen (Oneto-One), eine Gruppe (One-to-Few) oder die Öffentlichkeit (One-to-Many) kommuniziert werden (Williams et al., 2013, S. 385). In Verbindung mit einer relativ hohen Anonymität wird hierdurch auch eine ungezwungene Konversation gefördert, beziehungsweise die Hemmschwelle für eine kritische oder sozial unerwünschte Äußerung zu einem Thema gesenkt. Dies begünstigt nicht nur die Tweet-Wahrscheinlichkeit und -häufigkeit eines Nutzers und somit den Datenumfang für Studien, sondern ermöglicht der Forschung auch Einblicke in die Psyche der Nutzer (Java et al., 2007; Zhao \& Rosson, 2009). Zudem beschränkt sich der Inhalt aufgrund der strengen Zeichenbegrenzung auf das Wesentliche: Statt langen Texten sind Nutzer gezwungen, in wenigen Sätzen Meinungen zu äußern oder Informationen zu vermittelt (boyd et al., 2010a).

\subsection{Datenstruktur}

Die Komprimierung des Inhalts auf konstante Textlängen und die hohe Standardisierung von Tweets durch feste Konventionen der Kommunikation ermöglichen eine einfache, automatisierte Auswertung der Inhalte auf Twitter (Marres \& Weltevrede, 2013). Über Hashtags können die wichtigsten Themen erfasst werden, Mentions, Replies und Followings geben Auskunft über die Vernetzung eines Nutzers, Retweets und Favorites zeigen die Zustimmung eines Nutzers zu einer Äußerung oder das Gefallen einer Meldung an (Bollen, Pepe et al., 2011; Pak \& Paroubek, 2010). Diese Informationen können schnell und ohne großen Aufwand aus Tweets extrahiert werden, da Twitter sie - wie auch Links zu Webseiten, Videos und Bilder - gesondert als Metadaten übermittelt.

Jedoch ist eine inhaltliche Analyse unter Umständen auch problematisch: Auf Twitter wird, wie in vielen anderen internetbasierten Diensten, die Sprache abgewandelt. Abkürzungen, Neologismen, vermischte Sprachen und ein unvollständiger Satzbau prägen die Konversation im Internet (Bifet \& Frank, 2010; Carter et al., 2013; Gottron \& Lipka, 2010). Dies kann, wie bereits in Kapitel 4.3.1 erwähnt, eine automatische Inhaltsanalyse durch Programme behindern oder zu Fehlinterpretationen führen. Ebenso ist die Richtigkeit der Nutzerangaben nicht verifiziert: Twitter-Nutzer können falsche Standortangaben machen, eine andere Sprache wählen oder fiktive Namen verwenden (Cheng et al., 2010; Orita \& Hada, 2009). 
Gerade die Tatsache, dass Tweets vor allem auf Englisch geschrieben werden (Semiocast, 2013), erschwert eine Zuordnung des Nutzers zu einer Nationalität.

Zudem fehlen wichtige, nutzerspezifische Merkmale wie das Alter oder das Geschlecht. Twitter verlangt diese Informationen nicht bei der Registrierung. Dies wiederum behindert eine mögliche Eingrenzung des zu beobachtenden Nutzerkreises für eine Erhebung. Es ist beispielsweise nicht möglich, nur Tweets von Bundesbürgerinnen zur Bundestagswahl zu erfassen, indem nur Tweets aus deutschen Städten in deutscher Sprache mit einem gewissen Hashtag gespeichert werden. Twitter ist eine globale Plattform und ist jederzeit von jedem Ort abrufbar. Somit eignen sich Twitter-Daten auch nur für global bezogene Analysen oder zumindest für solche, bei denen sozidemographische Merkmale, wie Alter, Geschlecht und Nationalität keine Bedeutung haben. Diese Einschränkung kann natürlich minimiert werden, indem Hashtags verwendet werden, die von sich aus nur von lokalem Interesse sind: \#tatort wird mit hoher Wahrscheinlichkeit vor allem von deutschsprachigen Personen verwendet und entsprechend selten in Asien oder Amerika. Dennoch besteht die Möglichkeit, dass auch Tweets erfasst werden, die für die Analyse keine Bedeutung haben.

In diesem Kontext ist eine Eingrenzung des Datensets auch deshalb schwierig, weil nicht jeder Nutzer zwangsläufig dasselbe Hashtag für ein Thema nutzt, geschweige denn überhaupt ein Hashtag verwendet. Sollen eher diffusere Themen, wie die Meinung zu einer Partei erfasst werden, fällt die Wahl des geeigneten Suchterms schwer. In diesem Fall empfehlen sich mehrere Abfragen mit unterschiedlichen Begriffen (Beispiel CDU: ,merkel“, ,cdu“, ,konservativen“”, ,christdemokraten" usw.).

Ein ähnliches Problem stellen Tweets und Replies dar, die zu einem Thema oder einer Aussage geschrieben werden, ohne dabei Begriffe zu enthalten, die eine Zuordnung zu einem Thema ermöglicht. Zum Beispiel: Nutzerin1 schreibt „der tatort ist sowas von langweilig“ und Nutzer2 antwortet: „@Nutzerin1: ich stimme dir zu!“‘. Ebenso können Aussagen, die Ironie oder Sarkasmus enthalten, aufgrund des nicht ersichtlichen Kontextes falsch interpretiert werden. Diese Zusammenhänge können nur bei einer qualitativen Analyse erfasst werden, die den Kontext von Tweets berücksichtigt. 


\subsection{Repräsentativität}

Twitter macht keine genauen Angaben zu Umfang und Vollständigkeit der durch die APIs übermittelten Daten, wie beispielsweise zu der Repräsentativität des Streaming API Samples. Das Unternehmen weist jedoch zumindest bei den REST APIs darauf hin, dass der Fokus dieser Schnittstellen auf der Übermittlung relevanter und nicht vollständiger Daten liegt (Twitter, Inc., 2015g). Das mag nicht nur daran liegen, dass Twitter den Zugriff auf vollständige Daten (über Firehose und den eigenen Datenhändler Gnip) als Einnahmequelle nutzt, sondern auch an den technologischen Herausforderungen bei der Bereitstellung von Echtzeit-Daten in einer ständig wachsenden Infrastruktur.

Bei der Betrachtung von Twitter-Daten sollte ferner immer berücksichtigt werden, dass die Nutzerzahl mit etwa 800 Millionen $^{30}$ weltweit zwar sehr hoch, die Zahl aktiver Nutzer mit 284 Millionen jedoch deutlich geringer ist, wovon wiederum etwa 30 Prozent US-Amerikaner sind (Semiocast, 2012). Dabei sind auch diese Werte nur eingeschränkt zuverlässig, da auch Semiocast die Herkunft der Accounts mittels der zur Verfügung stehenden Daten schätzen muss. Dennoch bleibt Twitter in vielen Ländern ein Nischen-Medium mit einem elitären Nutzerkreis: 2013 nutzten in Deutschland etwa 7 Prozent Twitter zumindest unregelmäßig, davon wiederum 7 Prozent täglich (Busemann, 2013, S. 398). 53 Prozent der Nutzer war zwischen 14 und 29 Jahren alt.

Dementsprechend ist die Repräsentativität von Twitter-Daten stark eingeschränkt. Norris (2001) spricht im Kontext der verzerrten Nutzung von OnlineMassenmedien auch von einem Digital Divide. Im Internet bildeten sich demnach vermehrt Netzeliten mit höherem sozioökonomischen Status beziehungsweise formaler Bildung, die neue Medien (wie Twitter) früher und intensiver nutzen sowie besser informiert seien. Die wenigen Studien, die sich mit der Demografie von Twitter-Nutzern beschäftigen, beschreiben den typischen Twitter-Nutzer als relativ jung (19-29 Jahre) mit höherem Bildungsgrad und etwas erhöhtem politischen Interesse im Vergleich zur Gesamtbevölkerung (Duggan, Ellison, Lampe, Lenhart, \& Madden, 2015; Gainous \& Wagner, 2014; Vaccari et al., 2013).

Somit sollte man immer beachten, dass die Zuverlässigkeit und Repräsentativität von Twitter-Daten eingeschränkt ist und beispielsweise mit Interviews oder Umfragen nicht verglichen werden kann. Nach Jungherr (2015, S. 25) erlaubt

\footnotetext{
${ }^{30}$ Twitter, Inc. nennt keine offiziellen Zahlen zu registrierten Benutzerkonten, sondern veröffentlicht nur die Zahl aktiver Nutzer. Ältere Schätzungen gehen von etwa 800 Millionen registrierten Konten aus (Edwards, 2013).
} 
Twitter zwar einen guten Einblick über die Veränderung von Absichten und Einstellungen von Nutzern, aber keine verlässlichen Rückschlüsse auf die allgemeine öffentliche Meinung. Eine Verlässlichkeit von Twitter-Inhalten ist nie vollständig gewährleistet: Weder die Echtheit eines (nicht-prominenten) Twitter-Accounts, noch die tatsächliche Meinung eines Nutzers oder die Echtheit einer verbreiteten Information können bestätigt werden (Gayo-Avello, 2012; Metaxas, Mustafaraj, \& Gayo-Avello, 2011).

\subsection{Datenverfügbarkeit}

Neben der mangelnden Repräsentativität von Twitter-Daten ist auch deren eingeschränkte Verfügbarkeit problematisch: Je nach Datenquelle können entweder zukünftige (Streaming API) oder vergangene (REST APIs) Tweets kostenlos abgefragt werden. Zudem gibt es noch eine Vielzahl von Drittanbietern, die ebenfalls unterschiedliche Einschränkungen hinsichtlich Zeitraum und Umfang haben. Letztlich wäre eine Vollständigkeit der Daten nur bei Zugriff auf die Firehose beziehungsweise bei Erwerb von Datenpaketen bei Gnip gewährleistet.

Zudem sind historische Tweets nach 6 bis 9 Tagen nur noch über einzelne $\mathrm{Ab}-$ fragen (Anhand der User- oder Tweet-ID) abrufbar. Bei sehr aufsehenerregenden Ereignissen, wie beispielsweise dem Finale der FIFA Fußball-WM der Männer, ist eine umfassende Erhebung von Tweets mit Hilfe der Streaming API nicht möglich, da sehr schnell das Bandbreiten-Limit von einem Prozent überschritten wird. Da die Suchergebnisse Search API nicht vollständig und zeitlich begrenzt sind, ist es für derartige Ereignisse sinnvoll, Daten nachträglich zu kaufen. Diese Einschränkungen behindern nicht nur die Forschung an sich, sondern erschweren auch eine Reproduzierbarkeit der Ergebnisse, da nicht gewährleistet ist, dass jede Abfrage mit gleichen Parametern identische Daten erzeugt (Gaffney \& Puschmann, 2014, S. 65).

Die Unvollständigkeit der Daten erzeugt noch ein weiteres Problem: Ähnlich wie bei der Analyse von wertenden Aussagen ist auch bei rein quantitativer Betrachtung der Kontext für eine Einordnung der Daten notwendig. Ohne das Wissen über das absolute Twitter-Volumen können, wie bereits in Kapitel 4.1.4 erwähnt, nur eingeschränkt Aussagen über die Bedeutung absoluter Zahlen getätigt werden. 


\subsection{Metriken und Methoden}

Obwohl sich die Wissenschaft bereits seit geraumer Zeit mit Twitter beschäftigt, fehlen noch immer allgemein gültige Standards hinsichtlich Metriken aber auch Methoden. Bruns und Stieglitz (2014, S. 69-70) thematisieren den Bedarf an akzeptierten Standards für die quantitative Analyse von Nutzer-Aktivitäten auf Twitter. Diese sollten flexibel ausgelegt sein, um sie bei unterschiedlichste Analyseeinheiten anwenden zu können: Von einzelnen Nutzern bis zu Gruppen, von spezifischen Hashtag-Analysen bis zu Ad-hoc-Analysen des gesamten Datenstroms. Diese Metriken würden die Vergleichbarkeit von Studien und deren Ergebnissen vereinfachen (Bruns \& Stieglitz, 2013, S. 3).

Mögliche Bereiche für Metriken wären nach Bruns und Burgess (2012) Hashtags, Nutzer und das Gesamtvolumen. Ein Beispiel wäre demnach das Verhältnis von aktiven zu wenig aktiven Nutzern, die ein Hashtag verwenden, um den Einfluss von sehr aktiven und vernetzten Nutzern bei der Verbreitung von Hashtags zu bestimmen. Hierfür fehlt dann allerdings wiederum die Definition von aktiven Nutzern. Nach der Sichtweise von Twitter, Inc. (2015k) sind Nutzer aktiv, wenn sie mindestens einmal im Monat den Twitter-Account verwenden (zum Lesen oder Schreiben). Huberman et al. (2008) setzten wiederum mindestens zwei Tweets voraus, wobei hier keine Angaben über den Zeitraum gemacht wurden. Cha et al. (2010) sahen eine aktive Nutzung erst ab einer Mindestzahl von 10 Tweets seit Erstellung eines Accounts. Ähnlich diffus ist die Definition von Meinungsführern (Lead Users).

Des Weiteren bemängeln Bruns und Stieglitz (2014) das Fehlen allgemeiner Informationen seitens Twitter über das absolute Twitter-Volumen zu einem Zeitpunkt. Zur richtigen Interpretation von Spitzen im Twitter-Volumen und zur Entwicklung sinnvoller Metriken (wie dem Anteil am Gesamtvolumen) bedarf es detaillierter Informationen über die (gesamte) Twitter-Aktivität. Damit könnten Ergebnisse gewichtet und verglichen werden. Problematisch ist hierbei auch, dass Tweet-Datensets, die über einen Suchbegriff erstellt werden, nur Tweets enthalten, in denen der Suchterm vorkommt. Dies wurde bereits im vorigen Kapitel angesprochen. Hier wäre es sinnvoll, wenn Twitter zumindest durch Replies verknüpfte Tweets in die Suchergebnisse mit einbeziehen würde, auch wenn diese den Suchterm im Text nicht enthalten.

Hierfür bedarf es aber einer Öffnung von Twitter für die Wissenschaft. Zwar gibt es bereits mit den Data Grants ein Programm für wissenschaftliche Nutzungszwecke (Krikorian, 2014) mit Vollzugriff auf alle erhobenen Twitter-Daten. Der Zugang ist aber auf sehr wenige, von Twitter ausgewählte Projekte beschränkt. 
Inwieweit sich in der Zukunft allgemein anerkannte Metriken etablieren und Twitter bei dieser Entwicklung positiv mitwirkt, bleibt offen.

Ähnlich wie bei den Metriken gibt es bisher auch in der Methodik keine Standards: der Erhebungszeitraum, die Suchterm-Logik (z.B. ein oder mehrere Hashtags, mit oder ohne Sprachfilter) und die Analysemethode variieren von Studie zu Studie. Selbst bei relativ strukturierten Vorgängen, wie der Sentiment-Analyse, gibt es eine Vielzahl an Ansätzen - von eigenen Skripten bis zu gängigen AnalyseTools wie beispielsweise WordStat (Williams et al., 2013). Die Tatsache, dass einige Autor/-innen weder die angewandte Analysemethode, noch das Vorgehen zur Erstellung des Datensatzes detailliert beschreiben, erschwert sowohl einen Vergleich, als auch die Reproduktion von Ergebnissen.

\subsection{Ethische und rechtliche Aspekte}

Neben technischen und wissenschaftlichen Problemen bestehen auch ethische und rechtliche Einschränkungen. Grundsätzlich muss jeder Nutzer - und somit auch Forschende - die rechtlichen Vereinbarungen von Twitter einhalten. Die rechtlichen Rahmenbedingungen verteilen sich auf vier Dokumente: Den allgemeinen Nutzungsbestimmungen Terms of Service (Twitter, Inc., 2015a), der Developer Policy (Twitter, Inc., 2014a), dem Developer Agreement (Twitter, Inc., 2014b) für Entwickler und der Datenschutzrichtlinie Privacy Policy (Twitter, Inc., 2015i). Zudem sollten Forschende immer auch die nationalen Datenschutzbestimmungen sowie - bei Verwendung von Drittanbieter-Programmen - weitere individuelle Bestimmungen berücksichtigen.

Fasst man diese Regelungen zusammen, lassen sich einige Einschränkungen für die Twitter-Forschung erkennen: Grundsätzlich darf die Datennutzung den wirtschaftlichen Interessen von Twitter nicht schaden. Twitter verbietet somit generell die Erstellung, Anreicherung und Verbreitung großer Datenbanken mit Tweets - und das auch für nicht-kommerzielle Zwecke (Beurskens, 2014). Dies ist allein Twitter und -im begrenzten Umfang - privilegierten Vertragspartnern vorbehalten. Zudem unterliegen größere Datenbanken mit (angereicherten) Nutzerdaten den jeweiligen nationalen Datenschutz-bestimmungen (Beurskens, 2014, S. 130). Dies erschwert die Weitergabe vorhandener Daten an andere Wissenschaftler/-innen und begrenzt dadurch die Möglichkeiten zur Reproduktion von Ergebnissen.

Es ergibt sich somit ein diffuses Bild: Einerseits stellen Twitter-Nutzer automatisch ihre Tweets der Öffentlichkeit zur Verfügung (dies ist der Sinn von Tweets). Diese Nachrichten können über die interne Suche oder Google gefunden 
werden. Jeder Nutzer sollte sich bewusst sein, dass prinzipiell jede Person die persönlich zur Verfügung gestellten Daten lesen kann. Andererseits behält sich Twitter die Rechte an diesen Tweets vor (zur Verarbeitung oder Weitergabe), erstellt aber gleichzeitig im Zuge einer Kooperation mit der amerikanischen Library of Congress ein öffentliches, historisches Archiv aller Tweets (Twitter, Inc., 2010). Folglich könnte man auch argumentieren, dass diese Twitter-Daten, die bereits veröffentlicht wurden, problemlos erneut (im Kontext einer Studie) verarbeitet und publiziert werden dürfen.

Da Twitter die Einhaltung seiner Regeln nur schwer kontrollieren kann, empfiehlt Beurskens (2014) eine pragmatische Vorgehensweise: Selbst erstellte Tweet-Sammlungen sollten nie veröffentlicht und der Zugriff auf Daten kontrolliert werden. Wäre eine Weitergabe von Daten für wissenschaftliche Zwecke notwendig, sollten diese immer anonymisiert werden, beispielsweise durch eine Pseudonymisierung oder ein Entfernen der entsprechenden, sensiblen Werte (name, screen_name, id). Eine Veröffentlichung/Weitergabe einzelner Tweets ist jedoch immer erlaubt und wird vor allem durch Medien ständig praktiziert

\subsection{Relevanz und Zukunft des Portals}

Insgesamt ist Twitter jedoch ein beachtenswertes Medium und eine nahezu unerschöpfliche Datenquelle für vielseitige Forschungsschwerpunkte. Das zeigt die Bandbreite an Studien: Die Meta-Analyse von Williams et al. (2013) analysierte über 1.000 wissenschaftliche Arbeiten, die im Zusammenhang mit Twitter stehen. Diese klassifizierten sie unter anderem in folgende Forschungsbereiche: Wirtschaft (v.a. PR, Marketing), Geografie (Bewegungsmuster, Aufenthaltsort), Gesundheit (Krankheiten), Kommunikation (Interpersonelle Kommunikation, Medien), Notfälle (Katastrophen), Klassifizierungen (Verhaltensmuster) und Sprache (Syntax, Semantik). Puschmann, Bruns, Mahrt, Weller und Burgess (2014, S. 426427) begründen die Relevanz von Twitter für die Forschung mit der zunehmenden Bedeutung des Mikroblogging-Dienstes. Twitter sei ein globales Phänomen, das stetig an Nutzern und Tweets und somit auch an verwertbaren Daten wächst. Die immer stärkere Einbettung in die Medienökologie (durch Politik, Journalismus, Unternehmen und Privatnutzer) unterstreiche die aufstrebende Rolle in der Gesellschaft und den wachsenden Einfluss auf die Gesellschaft.

Dennoch ist die mittel- bis langfristige Zukunft von Twitter offen. Während die Zahl der monatlich aktiven Nutzer bis 2015 konstant wuchs, sank der Wert im vierten Quartal 2015 erstmals um 2 Millionen auf nun 305 Millionen (Twitter, 
Inc., 2016). Zudem verbuchte das Unternehmen in den letzten Jahren erhebliche Verluste: Allein 2014 hatte Twitter einen Netto-Verlust von 578 Millionen USDollar (Twitter, Inc., 2015j).

Inwieweit das die zukünftige Architektur von Twitter hinsichtlich Funktionen und Zugriffsmöglichkeiten beeinflusst, ist noch unklar. Möglich wären restriktivere Datenzugänge (dies zeigt sich bereits in der Konzentration auf Gnip als alleinigen Daten-händler) - nicht nur für kommerzielle Nutzer, sondern auch für die Forschung. Zudem plant Twitter eine Ausweitung der Werbeanzeigen durch bessere Vermarktungsfunktionen auf der Plattform (Bragdon, 2015). Anfang 2016 verkündete Twitter mehrere tiefgreifende Veränderungen in der Systematik der Plattform. Zum einen überarbeitet das Unternehmen den Algorithmus zur Darstellung von Tweets im Feed: Anstelle einer streng chronologischen Auflistung von Tweets werden nun Tweets von Followees vorgefiltert und nach individueller Bedeutsamkeit und Popularität an den Anfang positioniert (Jahr, 2016). Diese Vorsortierung ist zunächst nicht verpflichtend, allerdings besteht die Möglichkeit, dass Twitter diese Funktion in absehbarer Zeit für eine lukrativere Positionierung von Werbung nutzen könnte.

Zum anderen wird überlegt, die Limitierung auf 140 Zeichen pro Tweet aufzuheben (Dorsey, 2016). Dies widerspräche der Grundidee von Twitter, Meldungen auf das Wesentliche zu konzentrieren und den Informationscharakter zu stärken. Die geringe Textlänge ist das wichtigste Merkmal der Kommunikation auf Twitter und ein Alleinstellungsmerkmal gegenüber anderen Plattformen, wie Facebook oder Instagram. Und nicht zuletzt ist die Zeichenbegrenzung ein wesentlicher Vorteil für die Textanalyse. Eine Erhöhung des Limits auf 10.000 Zeichen, wie es momentan geplant wird, verändert letztlich nicht nur die Kommunikationsmuster und -inhalte, sondern auch die Möglichkeit zur Datenanalyse.

Beiden Mitteilungen führten zu einer großen Resonanz bei Nutzern und Medien. Über das Hashtag \#RIPTwitter kritisierten viele Nutzer die Abkehr von den Grundprinzipien des Dienstes (Steiner, 2016). Die langfristige Reaktion der Nutzer (z.B. als Einbruch der Nutzerzahlen) wird sich in der Zukunft zeigen. Letztlich ist das Internet sehr schnelllebig: Regelmäßig entstehen und verschwinden OnlineKommunikationsportale. Möglich wäre also ein ähnlich rapider Bedeutungsverlust von Twitter - auch aufgrund disruptiver Innovationen - wie er bei den sozialen Netzwerken StudiVZ und MySpace nach dem Erfolg von Facebook zu beobachten war.

\footnotetext{
Open Access Dieses Kapitel wird unter der Creative Commons Namensnennung - Nicht kommerziell 4.0 International Lizenz (http://creativecommons.org/licenses/ by-nc/4.0/deed.de) veröffentlicht, welche für nicht kommerzielle Zwecke die Nutzung, Vervielfältigung, Bearbeitung, Verbreitung und Wiedergabe in jeglichem Medium und Format erlaubt, sofern Sie den/die ursprünglichen Autor(en) und die Quelle ordnungsgemäß nennen, einen Link zur Creative Commons Lizenz beifügen und angeben, ob Änderungen vorgenommen wurden.

Etwaige Abbildungen oder sonstiges Drittmaterial unterliegen ebenfalls der genannten Creative Commons Lizenz, sofern sich aus der Abbildungslegende oder der Quellreferenz nichts anderes ergibt. Sofern solches Drittmaterial nicht unter der genannten Creative Commons Lizenz steht, ist eine Vervielfältigung, Bearbeitung oder öffentliche Wiedergabe nur mit vorheriger Zustimmung des betreffenden Rechteinhabers oder auf der Grundlage einschlägiger gesetzlicher Erlaubnisvorschriften zulässig.
} 